\title{
Simulation Bounds FOR EQuivalence VERIFICATION OF ARITHMETIC DATAPATHS WITH FINITE WORD-LENGTH OPERANDS *
}

\author{
Namrata Shekhar, Priyank Kalla \\ Electrical \& Computer Engineering Department \\ University of Utah, Salt Lake City, UT-84112 \\ \{shekhar,kalla\}@eng.utah.edu
}

\author{
M. Brandon Meredith, Florian Enescu \\ Department of Mathematics \& Statistics \\ Georgia State University, Atlanta, GA-30303 \\ \{fenescu@mathstat,mmeredith5@student\}.gsu.edu
}

\begin{abstract}
This paper addresses simulation-based verification of highlevel descriptions of arithmetic datapaths. Instances of such designs are commonly found in DSP for audio, video and multimedia applications, where the word-lengths of input/output bit-vectors are fixed according to the desired precision. Initial descriptions of such systems are usually specified as Matlab/C code. These are then automatically translated into behavioural/RTL descriptions (HDL) for subsequent hardware synthesis.

In order to verify that the initial Matlab/C model is bit-true equivalent to the translated RTL, how many simulation vectors need to be applied? This paper explores results from number theory and commutative algebra to show that exhaustive simulation is not necessary for testing their equivalence. In particular, we derive an upper bound on the number of simulation vectors required to prove equivalence or identify bugs. These vectors cannot be arbitrarily generated; we determine exactly those vectors that need to be simulated. Extensive experiments are performed within practical CAD settings to demonstrate the validity and applicability of these results.
\end{abstract}

\section{INTRODUCTION}

Increasing size and complexity of digital systems has resulted in a vast array of formal verification techniques which operate at different levels of abstraction. In spite of many such advances, simulation-based validation has remained an important method for ensuring functional correctness during various stages of the design cycle. Fig. 1 describes a typical design flow for arithmetic datapath intensive (signal-processing) applications, along with the context in which the verification problem appears.

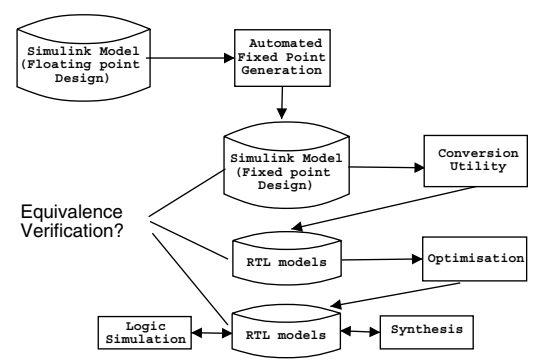

Fig. 1. The Equivalence Verification problem: Matlab to RTL design flow.

Initial algorithmic specifications (such as MATLAB models) of most signal processing applications involve data representation using floating-point formats. However, they are often implemented with fixed-point architectures, where the required precision dictates the bit-vector sizes of the variables. Various automated tools exist for this translation [1]. For synthesis and optimization purposes, these high-level descriptions may be subsequently converted to HDL using automatic utilities [2]

${ }^{*}$ This work is sponsored in part by NSF CAREER grant CCF-546859 and NSF grant CCF-515010.
[3]. Design optimization may be further achieved by applying high-level synthesis and restructuring operations on the translated RTL model [4] [5]. It is required to show that the translated and optimized RTL models are bit-true equivalent to the fixed-point specification.

Simulation is extensively used to validate the input-output behavior of the original model (at the MATLAB, C level); say to validate the pass-band of a filter. These vectors can then also be applied at the RT-level. Validation of the Matlab model is fast, even with a large number of test vectors, since it is compiled-code simulation. However, simulating the RTL model with the same set of vectors is generally slow. In this regard, this paper derives an important result related to simulation-based verification of high-level descriptions of arithmetic datapaths. In particular, we show that:

1. Exhaustive simulation is not always necessary to verify equivalence or to find bugs;

2. An upper bound is derived for the maximum number of test vectors required for this purpose; and

3. Which vectors to choose for simulation.

Further, we model such arithmetic-intensive design descriptions as polynomial functions. Moreever, the word-lengths of the variables are usually predetermined and fixed. For correct modeling, we need to account for the effect of the fixed bitwidths of the input/output variables. Hence, the polynomial functions are computed over finite-integer rings where the ring cardinality corresponds to the datapath size. We then apply concepts from number theory to systematically establish the claims mentioned above.

Let us motivate this issue using a practical example and put our contribution in perspective.

\section{A. An Example Application}

Given two degree- $k$ polynomials $F_{1}(x)$ and $F_{2}(x)$, their coefficients can be represented as $(k+1)$-length vectors $A=$ $\left(a_{0}, \ldots, a_{k}\right)$ and $B=\left(b_{0}, \ldots, b_{k}\right)$. Computing the convolution of such vectors results in another $k+1$ vector, according to:

$$
c_{i}=\sum_{j=0}^{k} a_{j} b_{i-j} \quad 0 \leq i \leq k
$$

where, $c_{i}$ is the $i^{t h}$ component in the vector $C$. This procedure, however, has a complexity of $O\left(n^{2}\right)$. It is well-known [6] [7] that convolution can be effectively implemented in hardware by:

1. Computing the DFT of vectors $A$ and $B$, 
2. Calculating their pairwise product; and finally,

3. Taking the inverse DFT of the result. In other words, the result vector $\left(c_{0}^{\prime}, c_{1}^{\prime}, \ldots, c_{k}^{\prime}\right)$ is computed as

$$
C^{\prime}=D F T^{-1}(D F T(A) \cdot D F T(B))
$$

This operation is shown in Fig. 2 for degree-3 polynomials. Such a 'divide-and-conquer' strategy results in a complexity of $O(n \cdot \log n)$, and is a popular way of implementing the convolution of two vectors.

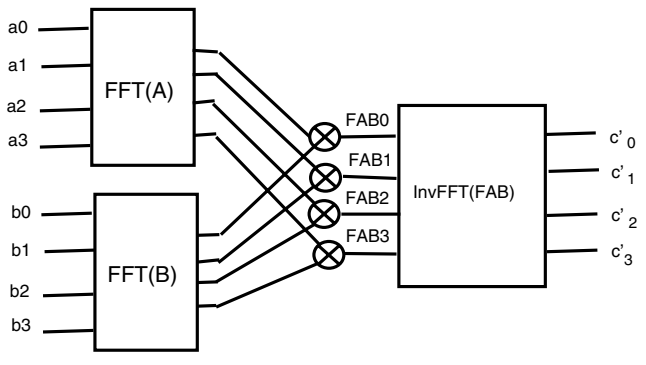

Fig. 2. Convolution of $A$ and $B$.

Suppose that we intend to verify that both implementations compute the same values, say, for coefficients $c_{0}$ (obtained via direct convolution) and $c_{0}^{\prime}$ (obtained via DFT-productInvDFT). Further, as is often the case, assume that the entire datapath word-length in both cases is fixed to a certain width, say 4-bits. Since each of the inputs $\left(a_{i}\right.$ and $\left.b_{i}, 0 \leq i \leq 3\right)$ can take values between $\left\{0, \ldots, 2^{4}-1\right\}$, is it necessary to simulate a total of $2^{4 * 8}$ test vectors to prove bit-true equivalence or to identify the presence of a bug?

This paper derives results which prove that in the above case: i) if the two designs are not equivalent (bug), a maximum of $6^{8}$ simulations are sufficient to capture the erroneous behavior; ii) if for these $6^{8}$ vectors, no bug is detected, then the designs are indeed equivalent. A method for generating these specific simulation vectors is also derived.

\section{B. Problem Modeling and Scope}

We model the arithmetic computations over bit-vectors as follows. Let $x_{1}, x_{2}, \ldots, x_{d}$ denote the $d$-variables (bit-vectors) in the design. Let $n_{1}, n_{2}, \ldots, n_{d}$ denote the size of the corresponding bit-vectors. Therefore, $x_{1} \in Z_{2^{n_{1}}}, x_{2} \in Z_{2^{n_{2}}}, \ldots, x_{d} \in$ $Z_{2^{n} d}$. Note that $Z_{2^{n_{i}}}$ corresponds to the finite set of integers $\left\{0,1, \ldots, 2^{n_{i}}-1\right\}$. Let $m$ correspond to the size of the output bit-vector $f$; hence, $f \in Z_{2^{m}}$. Subsequently, we model the arithmetic datapath computation as a polynomial function (or polyfunction) from $Z_{2^{n_{1}}} \times Z_{2^{n_{2}}} \times \cdots \times Z_{2^{n_{d}}}$ to $Z_{2^{m}}$ [8]. Here $Z_{a} \times Z_{b}$ represents the Cartesian product of $Z_{a}$ and $Z_{b}$. In other words, the computation is modeled as a multi-variate polynomial $F\left(x_{1}, x_{2}, \ldots, x_{d}\right) \% 2^{m}$, where each $x_{i} \in Z_{2^{n_{i}}}$ and $F$ is computed $\% 2^{m}$. The equivalence problem then corresponds to checking the congruence of two polynomials: $F \equiv G \% 2^{m}$.

Note: Our approach is applicable to high-level descriptions of bit-vector arithmetic that can be easily abstracted as polyfunctions. For this reason, our approach cannot be used to verify a behavioural RTL model against its gate-level netlist.
The verification problem of Fig. 1 has seen a lot of interest recently in [9] [10] [11] [12] [13]. The works of [12] [13] use the same polynomial function model to derive a symbolic approach to prove/disprove equivalence of arithmetic datapaths. However, these works are restricted inasmuch as they can only provide a "yes/no" answer to the equivalence check. They cannot provide an error trace when bugs are detected. Moreover, our results also have implications on the applicability of the fundamental theorem of algebra which has been used in hardware design and verification [9] [10] [11], as discussed below.

\section{Bit-Vector Arithmetic versus the Fundamental Theorem of Algebra}

Lemma I.1: Let $P(x)$ be a degree- $k$ uni-variate polynomial. If $P(x)=0$ for $(k+1)$ distinct values of $x$, then all the coefficients of $P(x)$ are zero.

The above lemma [14] is based on the fundamental theorem of algebra [15]. This theorem states that a degree- $k$ univariate polynomial $P(x)$ has exactly $k$ complex roots, unless all its coefficients are zero. Since integers are a special case of complex numbers, this theorem holds for the set $Z$ as well. The work of [10] used this result for equivalence verification by modeling arithmetic datapaths as polynomials, and showed that $k+1$ vectors were sufficient to prove equivalence of any given degree- $k$ polynomials. It was later extended in [14] to be applicable to multi-variate polynomials as well, and was further applied to reduce the complexity of model-checking. Also, [9] used the same concepts for polynomial extraction for high-level synthesis purposes.

However, the above results are only relevant in unique factorization domains (UFDs), such as the set of real numbers $(R)$, the set of integers $(Z)$, finite fields $\left(Z_{p}, G F\left(p^{n}\right), p=\right.$ prime $)$ and so on. Finite-word-length arithmetic does not correspond to UFDs. Since a bit-vector of size $m$ represents integer values reduced $\% 2^{m}$, bit-vector arithmetic corresponds to a ring (and not a field) $Z_{2^{m}}$, which contains zero-divisors. Therefore, when the bit-vector sizes are accounted for in our model, polynomials are computed modulo an integer power of 2. Consequently, factorization is not unique as shown for the polynomial $F(x)=x^{2}+6 x$ below.

$$
\begin{aligned}
x^{2}+6 x & =x(x+6) \% 2^{3} \\
& =(x+4)(x+2) \% 2^{3}
\end{aligned}
$$

The polynomial $F(x)$ has a degree $k=2$, but can be factorized in two non-unique ways; corresponding to four unique roots. In such cases, Lemma I.1 does not hold; simulating for $k+$ 1 values such as $x=0,2,4$, does show $F=0$ but that does not mean that all the coefficients of $F(x)$ are zero. Therefore, simulating for only $k+1$ vectors is insufficient for verification.

Clearly, for finite-word-length bit-vector arithmetic, properties of these class of rings (of the type $Z_{2^{m}}$ ) need to be investigated further for simulation-based verification. This paper explores results for polynomial functions over such finite integer rings and develops solutions to such problems with applications in simulation-based verification of arithmetic datapaths. 


\section{Paper Organization}

This paper is organized as follows: The next section reviews related work in simulation-based verification. Section III covers preliminary concepts and background material regarding polynomial functions and finite ring theory. Section IV describes the proposed results for univariate polynomials and provides the mathematical foundation for their support. These results are extended for multi-variate computations as well. Finally, Section V describes the experimental setup and results, while Section VI concludes the paper.

\section{PREVIOUS WORK}

Bryant, in [16] [17], used three-valued logic simulation to reduce the required number of simulation vectors for circuit verification. Subsequently, [18] incorporated some of these techniques into their framework, which provided a method for design verification at different levels of abstraction. Brand [19] proposed exploiting information from the design specification to significantly reduce the complexity of simulation. Clarke $e t$ $a l$. further researched the problem of specifications and generators in [20]. However, BDDs were used to demonstrate a practical approach to this problem in the SimGen project [21]. Later on, Shimizu et al. [22] [23] automated this approach to verify large industrial designs. The above methods are focused towards generating the appropriate number of test vectors to ensure sufficient verification coverage. Our approach, on the other hand, applies polynomial methods that make exhaustive simulation unnecessary for arithmetic datapaths.

An algebraic approach to reducing the test vector set was proposed in [10], and later extended in [11]. These works model the given datapaths as polynomials and apply the fundamental theorem of algebra to verify the descriptions. Recently, [14] extended the theorem to be applicable to multivariate polynomials as well. However, as mentioned earlier, these results do not always hold over the more practical cases of finite word-length bit-vector arithmetic.

The works which come closest to ours have been presented in [24][13]. Both approaches model the given datapath as a polynomial function over a system of finite integer rings. [24] proves equivalence of fixed-size datapaths by using canonical representations of polynomials over finite rings. The concept of vanishing polynomials is used in [13] to derive a symbolic approach to test equivalence of polynomial functions. Both approaches utilize some form of algebraic simplification, which suffers from the well-known intermediate-expression swell problem [25]. In addition, these techniques cannot provide an error trace whenever non-equivalence is detected.

This paper reinterprets the polynomial function model and extends the concepts presented in [8] to derive a novel solution for simulation-based verification of high-level descriptions of arithmetic datapaths. The next two sections cover some preliminary concepts, and then derive the theoretical contributions of this paper. Practical application of our work is subsequently demonstrated.

\section{PRELIMINARIES}

In what follows, $Z$ corresponds to the set of integers and $Z_{2^{m}}$ to the finite set of integers $\left\{0,1, \ldots, 2^{m}-1\right\}$, over which addition and multiplication are closed. $Z_{2^{m}}[x]$ denotes the ring of univariate polynomials over the variable $x$, with coefficients from $Z_{2^{m}}$. We use the following multi-index notation: $\mathbf{k}=<k_{1}, k_{2}, \ldots, k_{d}>$ are the (non-negative) degrees corresponding to the $d$ input variables $\mathbf{x}=\left\langle x_{1}, x_{2}, \ldots, x_{d}\right\rangle$, respectively. Also, $n_{1}, n_{2}, \ldots, n_{d}$ and $m$ are the input and output bit-vector sizes. Subsequently, we represent the RTL computations as polyfunctions from $Z_{2^{n_{1}}} \times Z_{2^{n_{2}}} \times \cdots \times Z_{2^{n} d}$ to $Z_{2^{m}}$, which are defined as [8]:

Definition III.1: A function $f$ from $Z_{2^{n_{1}}} \times Z_{2^{n_{2}}} \times \ldots \times Z_{2^{n} d}$ to $Z_{2^{m}}$ is said to be a polynomial function (or polyfunction) if it is represented by a polynomial $F \in Z\left[x_{1}, x_{2}, \ldots, x_{d}\right]$; i.e. $f\left(x_{1}, x_{2}, \ldots, x_{d}\right) \equiv F\left(x_{1}, x_{2}, \ldots, x_{d}\right)$ for all $x_{i}=0,1, \ldots, 2^{n_{i}}-1$; $i=1,2, \ldots, d$. Here, $\equiv$ denotes congruence $\bmod 2^{m}$.

Example III.1: Let $f: Z_{2^{1}} \times Z_{2^{2}} \rightarrow Z_{2^{3}}$ be a polyfunction in two variables $\left(x_{1}, x_{2}\right)$, defined as:

$f(0,0)=1, f(0,1)=3, f(0,2)=5, f(0,3)=7, f(1,0)=1$, $f(1,1)=4, f(1,2)=1, f(1,3)=0$.

Then, $f$ is a polyfunction representable by $F=1+2 x_{2}+x_{1} x_{2}{ }^{2}$, since $f\left(x_{1}, x_{2}\right) \equiv F\left(x_{1}, x_{2}\right) \% 2^{3}$ for $x_{1}=0,1$ and $x_{2}=0,1,2,3$.

It is possible for a polynomial with non-zero coefficients to vanish on such mappings, in which case it represents a nil polyfunction. Such polynomials are often called vanishing polynomials.

Example III.2: Consider the function $f\left(x_{1}, x_{2}\right): Z_{2} \times Z_{2^{2}} \rightarrow$ $Z_{2^{3}}$ represented by the polynomial $F=4 x_{1} x_{2}^{2}+4 x_{1} x_{2}$. While $F$ has non-zero coefficients, $F \% 8 \equiv 0, \forall x_{1} \in Z_{2}, \forall x_{2} \in Z_{4}$.

Properties of such polynomials have been extensively studied in number theory and commutative algebra [26] [27] [8]. We summarize some of these results in the context of our work.

\section{A. On Vanishing Polynomials}

In the ring $Z_{2^{m}}$, let $\lambda$ denote the least value such that $2^{m} \mid \lambda$ !. This value is called the Smarandache function of $2^{m}$ or $S F\left(2^{m}\right)$. For example, $S F\left(2^{3}\right)=4$ as 8 divides 4 ! (= $4 \times 3 \times 2 \times 1=8 \times 3$ ). Note that 8 does not divide 3 !, and hence the least $\lambda$ in question $=4$.

The significance of the above concept can be explained as follows. Consider the ring $Z_{2^{3}}$, where $\operatorname{SF}\left(2^{3}\right)=4$ since $2^{3} \mid 4$ !. Consequently, any integer that can be factored into a product of (at least) $\lambda=4$ consecutive numbers will be divisible by $2^{3}$ and vanish in $Z_{2^{3}}$. Now consider a polynomial $f(x)$ in the ring $Z_{2^{3}}$, such that $2^{3} \mid f(x)$. Therefore, if $f$, evaluated at $x$, can be represented as the product of 4 consecutive numbers (depending on $x$ ), then $f$ would vanish in $Z_{2^{3}}$. A natural example of such a polynomial is: $(x)(x-1)(x-2)(x-3)$. In this regard, Chen [27] proposed a set of monic polynomials, $Y_{k}(x)$, where each $Y_{i}(x)$ represents (in polynomial form) a product of $i$ consecutive numbers in $x$. More formally, we have the following definition and corresponding result:

Definition III.2: Falling factorials of degree $k$ are defined as:

- $Y_{0}(x)=1$

- $Y_{1}(x)=x$ 
- $Y_{2}(x)=x(x-1)$

- $Y_{k}(x)=x \cdot(x-1) \cdots(x-k+1)$

In the case of $d$ variables, $\mathbf{Y}_{\mathbf{k}}(\mathbf{x})=\prod_{i=1}^{d} Y_{k_{i}}\left(x_{i}\right)$.

Lemma III.1: Any polynomial in $Z_{2^{m}}[x]$ that can be expressed as a factor of $Y_{\lambda}(x)$ will be divisible by $2^{m}$ and vanish.

When such a factorization is not feasible, it is still possible for a given polynomial to $\equiv 0 \% 2^{m}$. In this regard, Singmaster [26] identified the constraints on the coefficients which would determine whether the polynomial in question would vanish. We state the following result.

Lemma III.2: The expression $c_{k} \cdot Y_{k}(x) \equiv 0$ in $Z_{2^{m}}[x]$ if and only if $\frac{2^{m}}{\left(k !, 2^{m}\right)} \mid c_{k}$;

where,

- $c_{k}$ is an arbitrary integer in $Z$,

- $Y_{k}(x)$ is as defined above,

- $\left(k !, 2^{m}\right)$ is the greatest common divisor (GCD) of $k$ ! and $2^{m}$ and

- $k$ is the degree of the expression $b \cdot Y_{k}(x)$, such that $k<\lambda$.

In other words, $c_{k} \equiv 0 \% \frac{2^{m}}{\left(k !, 2^{m}\right)}$ implies that $c_{k} \cdot Y_{k}(x) \equiv 0$.

Example III.3: Let $F(x)=4 x^{2}-4 x$ over $Z_{2}[x]$. Note that $F(x)=4(x)(x-1)=4 \cdot Y_{2}(x)$. Therefore, in this case $k=2$ and $c_{2}=4$. Also, $c_{2} \equiv 0 \% \frac{2^{3}}{\left(2 !, 2^{3}\right)}(=4)$. Because the above condition is satisfied, $F(x) \% 2^{3} \equiv 0$. Note if $c_{2}$ were replaced by 3 , then $F(x)=3(x)(x-1)$ would not be a vanishing polynomial as $3 \neq 0 \% \frac{2^{3}}{\left(2 !, 2^{3}\right)}$.

Based on the above concepts, Singmaster proposed a unique representation for any univariate vanishing polynomial over $Z_{2^{m}}$; i.e. $F(x) \equiv 0 \% 2^{m}$ if and only if it can be represented as:

$$
F(x)=Q_{\lambda}(x) Y_{\lambda}(x)+\sum_{k=0}^{\lambda-1} c_{k} Y_{k}(x)
$$

where

- $c_{k} \in Z$ is a multiple of $\frac{2^{m}}{\left(2^{m}, k !\right)}$

- $Q_{\lambda}(x) \in Z$ is any arbitrary polynomial

- $Y_{\lambda}(x)$ represents the product of $\lambda$ consecutive numbers.

Example III.4: Let us explain this result using the previous example. Consider $F(x)=4 x^{2}-4 x$ over $Z_{2^{3}}$. Here, $\lambda=S F\left(2^{3}\right)=4$. However, $F$ cannot be factored into $Y_{4}(x)$, therefore $Q_{4}=0$. Similarly, $c_{3}=0$, as $F$ cannot be factored into $Y_{3}(x)$. Now, $F$ can be factored using $Y_{2}(x)$, implying $c_{2}=4$ which is a multiple of $\frac{2^{3}}{\left(2^{3}, 2 !\right)}=4$. Therefore, corresponding to Eqn. $3, F(x)=4 \cdot Y_{2}(x) \equiv 0 \% 2^{3}$.

The above results were extended by Chen [8] for multivariate polynomials in $d$ variables over $Z_{2^{n_{1}}} \times Z_{2^{n_{2}}} \times \ldots \times Z_{2^{n_{d}}}$ to $Z_{2^{m}}$.

\section{B. Multivariate Vanishing Polynomials}

The notion of exploiting the value $\lambda$ for divisibility of polynomials was extended by [8] to expressions in multiple variables. Chen consequently, defines $\mu_{i}$, which is the minimum of $\lambda$ and $2^{n_{i}}$, for $1 \leq i \leq d$.
Lemma III.3: If a polynomial $F(\mathbf{x})$ over $Z_{2^{n_{1}}} \times Z_{2^{n_{2}}} \times \ldots \times$ $Z_{2^{n} d}$ to $Z_{2^{m}}$ can be factorized into a product of $\mu_{i}$ consecutive numbers in at least one of the variables $x_{i}$, then it vanishes $\% 2^{m}$.

Example III.5: Let $f: Z_{2^{1}} \times Z_{2^{2}} \rightarrow Z_{2^{3}}$ and its corresponding polynomial be $F=x_{1}^{2} x_{2}-x_{1} x_{2}$. Here, $\lambda=4$, degree $\left(x_{1}\right)=$ $k_{1}=2$ and degree $\left(x_{2}\right)=k_{2}=1$. Note that $\mu_{1}=\min \left\{2^{n_{1}}, \lambda\right\}=$ $\min \left\{2^{1}, 4\right\}=2=k_{1}$ (the condition in Lemma III.3 is satisfied) and $\mu_{2}=\min \left\{2^{n_{2}}, \lambda\right\}=\min \left\{2^{2}, 4\right\}=4>k_{2}$. Thus, $F$ can be written as a product of $m u_{1}$ consecutive numbers in $x_{1}$ :

$$
\begin{aligned}
x_{1}^{2} x_{2}-x_{1} x_{2} & \equiv x_{1}\left(x_{1}-1\right) x_{2} \\
& \equiv Y_{<2,1>}\left(x_{1}, x_{2}\right) \\
& \equiv 0 \% 2^{3}
\end{aligned}
$$

Lemma III.4: The expression $c_{\mathbf{k}} \cdot \mathbf{Y}_{\mathbf{k}} \equiv 0$ if and only if $\frac{2^{m}}{\left(2^{m}, \prod_{i=1}^{d} k_{i} !\right)} \mid c_{\mathbf{k}} ;$ where:

- $c_{\mathbf{k}} \in Z$ is an arbitrary integer;

- $\mathbf{k}=<k_{1}, \ldots, k_{d}>\in Z^{d}$ such that $k_{i}<\mu_{i}, \forall i=1, \ldots, d$;

- $\mathbf{Y}_{\mathbf{k}}$ is from Def. III.2 and

- $\left(2^{m}, \prod_{i=1}^{d} k_{i} !\right)$ is the greatest common divisor (GCD) of $2^{m}$ and $\prod_{i=1}^{d} k_{i} !$.

Similar to the univariate case, this result is equivalent to $c_{\mathbf{k}} \equiv$ $0 \% \frac{2^{m}}{\left(2^{m}, \prod_{i=1}^{d} k_{i} !\right)}$ implies $c_{\mathbf{k}} \cdot \mathbf{Y}_{\mathbf{k}} \equiv 0$.

Example III.6: Consider the polynomial $F=4 x_{1} x_{2}^{2}+4 x_{1} x_{2}$ corresponding to $f\left(x_{1}, x_{2}\right): Z_{2^{1}} \times Z_{2^{2}} \rightarrow Z_{2^{3}}$. We can use Lemma III.4 to prove that $f$ is a nil polyfunction. Here, $\lambda=4 ; \mu_{1}(2)=\min \{2,4\}=2, \mu_{2}(4)=\min \{4,4\}=4$. Also, $\mathbf{k}=<k_{1}, k_{2}>=<1,2>$ corresponds to the highest degrees of $x_{1}, x_{2}$. Moreover, $\prod_{i=1}^{2} k_{i} !=1 ! \cdot 2 !=2$.

$$
\begin{aligned}
F & \equiv 4 x_{1} x_{2}^{2}+4 x_{1} x_{2} \\
& \equiv 4 \cdot x_{1} \cdot x_{2} \cdot\left(x_{2}-1\right) \\
& \equiv c_{<1,2>} \cdot Y_{<1,2>}\left(x_{1}, x_{2}\right) \\
& \equiv 0 \% 2^{3}
\end{aligned}
$$

because $c_{<1,2>}=4 \equiv 0 \% \frac{8}{(8,1 ! \cdot 2 !)}$.

The canonical representation for a multivariate vanishing polynomial is shown below. $F\left(x_{1}, x_{2}, \ldots, x_{d}\right) \equiv 0 \% 2^{m}$ if and only if it can be represented as:

$$
F(\mathbf{x})=Q_{\mu}(\mathbf{x}) Y_{\mu}(\mathbf{x})+\sum_{\mathbf{k}} c_{\mathbf{k}} Y_{\mathbf{k}}(\mathbf{x})
$$

where

- $\mu_{i}=\min \left\{2^{n_{i}}, \lambda\right\}$

- $\mu=<\mu_{1}, \mu_{2}, \ldots, \mu_{d}>$

- $Y_{\mu}(\mathbf{x})=Y_{\mathbf{k}}(\mathbf{x})$ for some $k_{i}=\mu_{i}$

- $c_{\mathbf{k}} \in Z$ is a multiple of $\frac{2^{m}}{\left(2^{m}, \mathbf{k} !\right)}$ where $\mathbf{k} !=\prod_{i=1}^{d} k_{i} !$.

Example III.7: Consider a polynomial $F(\mathbf{x})=x_{1}^{2}+7 x_{1}+$ $4 x_{1} x_{2}^{2}+4 x_{1} x_{2}$ for $f: Z_{2} \times Z_{2^{2}} \rightarrow Z_{2^{3}}$. Here, $\lambda=4$. Further, $\mu_{1}=\min \{2, \lambda\}=2 ; \mu_{2}=\min \left\{2^{2}, \lambda\right\}=4 . \quad F\left(x_{1}, x_{2}\right)$ can be written as follows: 


$$
\begin{aligned}
F\left(x_{1}, x_{2}\right) & \equiv x_{1}\left(x_{1}-1\right)+4 \cdot x_{1} \cdot x_{2} \cdot\left(x_{2}-1\right) \\
& \equiv Y_{<2,0>}\left(x_{1}, x_{2}\right)+c_{<1,2>} Y_{<1,2>}\left(x_{1}, x_{2}\right) \\
& \equiv 0 \% 2^{3}
\end{aligned}
$$

Here, $Y_{<2,0>}\left(x_{1}, x_{2}\right)$ represents a product of $\mu_{1}$ consecutive numbers in $x_{1}$. Also, $c_{<1,2>}=4$ is a multiple of $8 /(8,1 ! \cdot 2$ ! $)=$ 4. Clearly, $F$ can be written in the form given by Eqn. 4 , and is thus a vanishing polynomial.

We now describe how some of these results can be used in the context of this work.

\section{THEORY}

\section{A. Univariate Polynomials}

Given a polynomial function $f(x)$ and its representative polynomial $F(x)$ over $Z_{2^{m}}$, we need to reinterpret $F$ in a way that would be more suitable for our purposes. In this context, we first define the forward difference operator $(\Delta)$ [28], which is a discrete analog to the derivative of a polynomial function.

Definition IV.1: Let $F(x)$ be a polynomial over $Z$.

$$
\begin{aligned}
(\Delta F)(x) & =F(x+1)-F(x) \\
\left(\Delta^{2} F\right)(x) & =(\Delta F)(x+1)-(\Delta F)(x) \\
\vdots & \\
\left(\Delta^{k} F\right)(x) & =\sum_{i=0}^{k}(-1)^{i}\left(\begin{array}{c}
k \\
i
\end{array}\right) F(k-i+x)
\end{aligned}
$$

Let us now apply the forward difference operator on a degree-2 polynomial in $x$.

Example IV.1: Let $F(x)=4 x^{2}+3 x$ be a polynomial in $Z$. Applying the $\Delta$ operator described in Def. IV.1, we get

$$
\begin{aligned}
(\Delta F)(x) & =F(x+1)-F(x) \\
& =4(x+1)^{2}+3(x+1)-\left(4 x^{2}+3 x\right) \\
& =8 x+7 \\
\left(\Delta^{2} F\right)(x) & =(\Delta F)(x+1)-(\Delta F)(x) \\
& =8(x+1)+7-(8 x+7) \\
& =8 \\
\left(\Delta^{3} F\right)(x) & =\left(\Delta^{2} F\right)(x+1)-\left(\Delta^{2} F\right)(x) \\
& =0
\end{aligned}
$$

We now state Newton's interpolation formula [29] based on the above definition. The proof for this formula is widely available in literature and is not reproduced here.

Definition IV.2: If $F(x)$ is a polynomial of degree $k$ with integral coefficients, then it can be written as

$$
F(x)=\sum_{i=0}^{k}\left(\Delta^{i} F\right)(0)\left(\begin{array}{l}
x \\
i
\end{array}\right)
$$

In other words, the given univariate polynomial can be expressed as an interpolation over a given set of tabulated points, which are in terms of the first value $(F(0))$ and the powers of the forward difference $(\Delta)$. It should be noted that the binomial term in Eqn. 6 can be expanded according to,

$$
\left(\begin{array}{l}
x \\
i
\end{array}\right)=\frac{x(x-1) \cdots(x-i+1)}{i !}=\frac{Y_{i}(x)}{i !}
$$

The numerator of this term, $Y_{i}(x)$, is the falling factorial from Def. III.2. Eqn. 6 can now be written as:

$$
F(x)=\sum_{i=0}^{k} \frac{\left(\Delta^{i} F\right)(0)}{i !} Y_{i}(x)
$$

Since $F(x)$ has integral coefficients, the expression $\frac{\left(\Delta^{i} F\right)(0)}{i !}$ is always an integer for $0 \leq i \leq k$. Note that $F(x)$ has a maximum of $(k+1)$ coefficients. This is illustrated in the following example:

Example IV.2: Consider the polynomial $F(x)=4 x^{2}+3 x$ in $Z$. Here, the degree of $F(x): k=2$ and $F$ can be expanded into $k+1=3$ terms. Using, Eqn. 8 and the values computed in Example IV.1, this function can be expressed as,

$$
\begin{aligned}
F(x)= & \sum_{i=0}^{2} \frac{\left(\Delta^{i} F\right)(0)}{i !} Y_{i}(x) \\
= & \frac{\left(\Delta^{0} F\right)(0)}{0 !} \cdot 1+\frac{\left(\Delta^{1} F\right)(0)}{1 !} \cdot x \\
& +\frac{\left(\Delta^{2} F\right)(0)}{2 !} \cdot x(x-1) \\
= & 7 x+4 x(x-1)
\end{aligned}
$$

Let us now apply the above interpretation to polynomials over finite integer rings, using the properties of vanishing expressions from Sec. III. Note that Eqns. 5 - 8 still hold, since the coefficients remain integral.

Let $F$ be any given polynomial corresponding to a polyfunction $f$ in $Z_{2^{m}}$. Let $\lambda$ denote the value of $S F\left(2^{m}\right)$. From Eqn. 3, $F$ can be written as,

$$
F(x)=Q_{\lambda}(x) Y_{\lambda}(x)+R(x)
$$

where, degree $(R(x))<\lambda$. For any arbitrary $Q_{\lambda} \in Z[x]$, $Q_{\lambda}(x) Y_{\lambda}(x)$ represents a multiple of $\lambda$ consecutive numbers and is $\equiv 0 \% 2^{m}$. The degree of $F(x)$ has now been reduced to $<\lambda$. Let us explain this representation with an example.

Example IV.3: Consider the polynomial $F(x)=x^{4}+3 x^{3}+$ $7 x^{2}+6 x$ over $Z_{2^{3}}$. The degree of $F(x)$ is 4 . We compute $\lambda=$ $S F\left(2^{3}\right)=4$, and divide $F(x)$ by $Y_{4}$ to represent it as:

$$
F(x)=Y_{4}(x)+x^{3}+4 x^{2}+4 x
$$

Here, $Q_{4}=1$ and $R(x)=x^{3}+4 x^{2}+4 x$. Now, $Y_{4}(x)$ represents a product of $\lambda$ consecutive numbers in $Z_{2^{3}}$, and evaluates to $0 \% 2^{3}$. Thus, $F(x)=x^{3}+4 x^{2}+4 x$, where the degree is now $3<\lambda$.

Now, using Newton's interpolation formula, we know that any function with integral coefficients can be represented according to Eqn. 8. Hence, we can now rewrite $F(x)$ as

$$
\begin{aligned}
F(x)=R(x) & =\sum_{k=0}^{\lambda-1} \frac{\left(\Delta^{k} R\right)(0)}{k !} Y_{k}(x) \\
& =\sum_{k=0}^{\lambda-1} b_{k} Y_{k}(x)
\end{aligned}
$$


since $\operatorname{deg}(R(x))<\lambda$. According to Lemma III.2, if all the coefficients $b_{k}$ of this expression reduce to 0 when computed $\% \frac{2^{m}}{\left(2^{m}, k !\right)}$, then $R(x)$ (and correspondingly, $F(x)$ ) vanishes in $Z_{2^{m}}$.

\section{B. Application to Equivalence Verification}

We now use the concepts stated in the previous sections to obtain the following result:

Theorem IV.1: Let $F_{1}(x)$ and $F_{2}(x)$ be two polynomials with coefficients in $Z_{2^{m}}$. To prove $F_{1}(x) \% 2^{m} \equiv F_{2}(x) \% 2^{m}$, it is sufficient to show that $F_{1}$ and $F_{2}$ are equivalent in $Z_{2^{m}}$ for any $\lambda$ consecutive values of $x$. Here, $\lambda$ is the least integer such that $2^{m} \mid \lambda !$.

Proof:

We use the the results described in previous sections to outline a systematic procedure as part of the proof for Theorem IV.1.

- Compute the value of $\lambda$. Express both $F_{1}(x)$ and $F_{2}(x)$ in the form of Eqn. 9. The degrees of $R_{1}(x)$ and $R_{2}(x)$ are now $<\lambda$.

- Represent $R_{1}(x)$ and $R_{2}(x)$ according to Newton's formula, given by Eqn. 11. The coefficients $\left(b_{k}\right)$ are computed for $0 \leq$ $k<\lambda$, and are unique.

- We know that if all the $b_{k}$ coefficients are $\equiv 0 \% \frac{2^{m}}{\left(2^{m}, k !\right)}$, then the polynomial vanishes. We can use this property to prove equivalence according to:

$$
\left(b_{k}\left(F_{1}\right)-b_{k}\left(F_{2}\right)\right) \equiv 0 \% \frac{2^{m}}{\left(2^{m}, k !\right)}
$$

or

$$
b_{k}\left(F_{1}\right) \% \frac{2^{m}}{\left(2^{m}, k !\right)} \equiv b_{k}\left(F_{2}\right) \% \frac{2^{m}}{\left(2^{m}, k !\right)}
$$

Thus, for each pair of corresponding coefficients $b_{k}\left(F_{1}\right)$ and $b_{k}\left(F_{2}\right)$, check if they are congruent modulo $\frac{2^{m}}{\left(2^{m}, k !\right)}$.

- If this check fails, then the polynomials are not equivalent. We halt the procedure. Else, we repeat the above step for all $b_{k}, 0 \leq k<\lambda$.

Following the above procedure, we need to compute and compare the $b_{k}$ values for at most $\lambda$ steps. Computation of each coefficient $b_{k}\left(F_{1}\right)$ (or $b_{k}\left(F_{2}\right)$ ) requires evaluation of $F_{1}(x)$ (or $F_{2}(x)$ ) according to Eqn. 11. This implies that $F_{1}(x)$ (or $\left.F_{2}(x)\right)$ is evaluated a maximum of $\lambda$ consecutive times.

Theorem IV.1 directly follows from this procedure.

Example IV.4: Consider the polynomials $F_{1}(x)=x^{5}+$ $15 x^{4}+5 x^{3}+x^{2}+2 x+8$ and $F_{2}(x)=x^{4}+10 x^{3}+3 x^{2}+2 x+8$ over $Z_{2^{4}}$. Here, $\lambda\left(2^{4}\right)=6$. To check their equivalence, we need to compare the values of $F_{1}(x)$ and $F_{2}(x)$ for any 6 consecutive values of $x$.

$$
\begin{array}{ll}
F_{1}(2)=8 ; & F_{2}(2)=8 \\
F_{1}(3)=0 ; & F_{2}(3)=8 \\
F_{1}(4)=0 ; & F_{2}(4)=0 \\
F_{1}(5)=0 ; & F_{2}(5)=0 \\
F_{1}(6)=0 ; & F_{2}(6)=0 \\
F_{1}(7)=0 ; & F_{2}(7)=0
\end{array}
$$

$F_{1}(3) \neq F_{2}(3) \% 2^{4}$; hence, these two polynomials are not the same in $Z_{2^{4}}$.

\section{Multivariate Polynomials}

The results of the previous section can be easily extended to arbitrary polynomials in $d$ variables.

Given any polynomial $F(\mathbf{x})$ corresponding to the polyfunction $f: Z_{2^{n_{1}}} \times Z_{2^{n_{2}}} \times \ldots Z_{2^{n} d} \rightarrow Z_{2^{m}}$, we use Eqn. 4 to represent it as

$$
F(\mathbf{x})=Q_{\mu}(\mathbf{x}) Y_{\mu}(\mathbf{x})+R(\mathbf{x})
$$

From Lemma III.3, $Q_{\mu}(\mathbf{x}) Y_{\mu} \equiv 0 \% 2^{m}$. This results in reducing the degree $\mathbf{k}$ of $F(\mathbf{x})$, such that $k_{i}<\mu_{i}$ for all $1 \leq i \leq d$.

Example IV.5: Let $F(\mathbf{x})=x_{1}^{2}+7 x_{1}+3 x_{1} x_{2}^{2}+4 x_{1} x_{2}$ over $Z_{2} \times Z_{2^{2}} \rightarrow Z_{2^{3}}$. Here, $\lambda=4$, and $\mu_{1}=\min \{2, \lambda\}=2$ and $\mu_{2}=\min \{4, \lambda\}=4$. We represent the polynomial as,

$$
F\left(x_{1}, x_{2}\right)=Y_{<2,0>}\left(x_{1}, x_{2}\right)+3 x_{1} x_{2}^{2}+4 x_{1} x_{2}
$$

In this case, $Y_{<2,0>}\left(x_{1}, x_{2}\right)$ is a product of $\mu_{1}=2$ consecutive numbers in $x_{1}$ and thus vanishes $\% 2^{3}$. Thus, $F(\mathbf{x})=R(\mathbf{x})=$ $3 x_{1} x_{2}^{2}+4 x_{1} x_{2}$ and has a degree $\mathbf{k}=<1,2>$, where $k_{1}=1<\mu_{1}$ and $k_{2}=2<\mu_{2}$.

We now define Newton's interpolation formula for multiple variables.

Definition IV.3: Let $F$ be a polynomial in $d$ variables $x_{1}, x_{2}, \ldots, x_{d}$ with degrees $\mathbf{k}=<k_{1}, k_{2}, \ldots, k_{d}>$. Then, Newton's formula can be written in multi-index notation as,

$$
\begin{aligned}
F(\mathbf{x}) & =\sum_{\mathbf{i} \leq \mathbf{k}}\left(\Delta^{\mathbf{i}} F\right)(\mathbf{0})\left(\begin{array}{l}
\mathbf{x} \\
\mathbf{i}
\end{array}\right) \\
& =\sum_{\mathbf{i} \leq \mathbf{k}} \frac{\left(\Delta^{\mathbf{i}} F\right)(\mathbf{0})}{i_{1} ! \ldots i_{d} !} \prod_{j=1}^{d} Y_{k_{j}}\left(x_{j}\right) \\
& =\sum_{\mathbf{i} \leq \mathbf{k}} \frac{\left(\Delta^{\mathbf{i}} F\right)(\mathbf{0})}{\mathbf{i} !} Y_{\mathbf{i}}(\mathbf{x})
\end{aligned}
$$

In the above, $\mathbf{i} \leq \mathbf{k}$ implies that $i_{1}<k_{1}, i_{2}<k_{2}, \ldots, i_{d}<k_{d}$. Applying this to $F(\mathbf{x})$, we get

$$
\begin{aligned}
F(x)=R(\mathbf{x}) & =\sum_{\mathbf{k} \leq \mu} \frac{\left(\Delta^{\mathbf{k}} R\right)(\mathbf{0})}{\mathbf{k} !} Y_{\mathbf{k}}(\mathbf{x}) \\
& =\sum_{\mathbf{k} \leq \mu} b_{\mathbf{k}} Y_{\mathbf{k}}(\mathbf{x})
\end{aligned}
$$

Again, note that maximum degree of $F(\mathbf{x})<\mu$. The coefficients $b_{\mathbf{k}}$ of the above formula are computed for the $\mathbf{k}=<$ $k_{1}, \ldots, k_{d}>$ vectors, where each $k_{i}=0, \ldots, \mu_{i}-1$. This corresponds to a maximum of $\prod_{i=1}^{d} \mu_{i}$ coefficients. We now state the following theorem.

Theorem IV.2: Let $F_{1}\left(x_{1}, \ldots, x_{d}\right)$ and $F_{2}\left(x_{1}, \ldots, x_{d}\right)$ be two polynomials over $Z_{2^{n}} \times Z_{2^{n}} \times \ldots Z_{2^{n} d}$ to $Z_{2^{m}}$. To prove/disprove their equivalence, it is sufficient to check for a total of $\prod_{i=1}^{d} \mu_{i}$ values, where $\mu_{i}$ is defined as the $\min \left\{2^{n_{i}}, \lambda\right\}$. Note that each 'value' is actually a $d$-tuple $\left\langle x_{1}, \ldots, x_{d}\right\rangle$, such that each $x_{i}$ corresponds to any $\mu_{i}$ consecutive values.

Proof:

The proof is based on the corresponding procedure for univariate polynomials, which is extended and reproduced below. 
- Compute the values $\mu_{1}, \ldots, \mu_{d}$. Express both $F_{1}(\mathbf{x})$ and $F_{2}(\mathbf{x})$ in the form of Eqn. 14. The degrees of $R_{1}(\mathbf{x})$ and $R_{2}(\mathbf{x})$ are now $<\mu$.

- Represent $R_{1}(\mathbf{x})$ and $R_{2}(\mathbf{x})$ according to Newton's formula, given by Eqn. 17. The coefficients $\left(b_{\mathbf{k}}\right)$ are computed for all $\mathbf{k}$, where $0 \leq k_{i}<\mu_{i}$, and are unique.

- We know that if all the $b_{\mathbf{k}}$ coefficients are $\equiv 0 \% \frac{2^{m}}{\left(2^{m}, \prod_{i=1}^{d} k_{i} !\right)}$, then the polynomial vanishes. As in the univariate case, we use this property to prove equivalence. Thus, for each pair of corresponding coefficients $b_{\mathbf{k}}\left(F_{1}\right)$ and $b_{\mathbf{k}}\left(F_{2}\right)$, check if they are congruent modulo $\frac{2^{m}}{\left(2^{m}, \prod_{i=1}^{d} k_{i} !\right)}$.

- If this check fails, then the polynomials are not equivalent. We halt the procedure. Else, we repeat the above step for all $b_{\mathbf{k}}$.

Following the above procedure, we need to compute and compare the $b_{\mathbf{k}}$ values for the tuples $\mathbf{k}=<k_{1}, k_{2}, \ldots, k_{d}>$, where each $k_{i}$ can take on any $\mu_{i}$ consecutive values. This requires evaluation of $F_{1}(x)$ (or $F_{2}(x)$ ) a maximum of $\prod_{i=1}^{d} \mu_{i}$ times.

Theorem IV.2 can be inferred from the above procedure.

Example IV.6: Let us now consider the polynomials $F\left(x_{1}, x_{2}\right)=x_{1} x_{2}^{3}+5 x_{1} x_{2}^{2}+2 x_{1} x_{2}$ and $F_{2}=x_{1}^{4} x_{2}+2 x_{1}^{3} x_{2}+$ $3 x_{1}^{2} x_{2}+x_{1} x_{2}^{3}+5 x_{1} x_{2}^{2}+4 x_{1} x_{2}$ over $Z_{2^{1}} \times Z_{2^{2}} \rightarrow Z_{2^{3}}$. Here, $\mu_{1}=2$ and $\mu_{2}=4$. We need to check if $F_{1} \equiv F_{2} \quad \% 2^{3}$. Therefore, we evaluate both polynomials for a maximum of $\mu_{1} \cdot \mu_{2}=8$ tuples. Here, $x_{1}$ can take any $\mu_{1}=2$ consecutive values and $x_{2}$ can take any $\mu_{2}=4$ consecutive values. Evaluating for $x_{1}=0,1$ and $x_{2}=0,1,2,3$ for $Z_{2^{3}}$, we get

$$
\begin{array}{rll}
F_{1}\left(x_{1}=0\right)=0 & ; & F_{2}\left(x_{1}=0\right)=0 \\
F_{1}\left(x_{1}=1 ; x_{2}=0\right)=0 & ; & F_{2}\left(x_{1}=1 ; x_{2}=0\right)=0 \\
F_{1}\left(x_{1}=1 ; x_{2}=1\right)=4 & ; & F_{2}\left(x_{1}=1 ; x_{2}=1\right)=4 \\
F_{1}\left(x_{1}=1 ; x_{2}=2\right)=0 & ; & F_{2}\left(x_{1}=1 ; x_{2}=2\right)=0 \\
F_{1}\left(x_{1}=1 ; x_{2}=3\right)=6 & ; & F_{2}\left(x_{1}=1 ; x_{2}=3\right)=6
\end{array}
$$

Since $F_{1}(\mathbf{x}) \equiv F_{2}(\mathbf{x})$ for 2 consecutive values of $x_{1}$ and 4 consecutive values of $x_{2}$, the two polynomials are equivalent.

\section{RESUltS}

Using the results from Theorems IV.1 and IV.2, we have been able to perform simulations over a number of designs collected from a variety of benchmark suites. The results are presented in Table I.

The first example is an image rejection computation. The phase-shift keying (PSK) [4] is used in digital communication. The polynomial filters [30] are Volterra models of polynomial signal processing applications. MIBench is a $9^{\text {th }}$-degree polynomial from [31]. The anti-aliasing function is commonly used in MP3 decoders and is from [4]. Horner polynomials [32] are commonly used in DSP - often implemented using multiplyadd-accumulate units. In [4], it was shown how computations by these MAC units can be extracted as polynomials in Horner's form. The last example is a vanishing polynomial of degree 10 .

Some of these designs were available as RTL code. The others were available as high-level specifications in MATLAB or $\mathrm{C}$ code. RTL code for these reference designs was automatically generated using the MATLAB Simulink and Filter Design toolboxes (particularly for the digital filter designs) [3]. Once the reference RTL descriptions were obtained, they were further optimized using techniques from [4] and [5]. In [4], application of high-level restructuring and symbolic algebrabased transformations was presented for high-level synthesis. These include factorization and expansion, tree-height reduction, etc. The recent work of [5] has derived a sequence of polynomial algebra based transformations to reduce the areacost of the implementation. This is achieved by modulating and segmenting the coefficients and subsequently removing algebraic redundancy (vanishing polynomials). These transformations were applied to the original RTL description to obtain functionally equivalent implementations.

Subsequently, the data-flow graphs for the original and optimized RTL descriptions were extracted using GAUT [33]. Traversing the DFGs from the inputs to the outputs, the polynomial representations were constructed. The datapath sizes of both inputs and outputs $\left(n_{1}, \ldots, n_{d}\right.$ and $\left.m\right)$ were also recorded. Using the proposed results, the maximum number of required test vectors was determined for all benchmarks. The descriptions were then simulated with these vectors to verify equivalence. We also wanted to analyze the performance of our approach in the presence of bugs. To verify that our algorithm can detect non-equivalence of designs, we experimented with some designs by arbitrarily changing one or more of the coefficients. In all cases, we were able to detect the erroneous values within the required number of simulations.

\section{A. Limitations of our approach}

Our approach works only on arithmetic datapaths whose functionality can be captured as polynomial functions. Hence, we cannot apply our technique to verify gate-level netlists. Also, many DSP systems implement some form of computation approximation, by incorporating various rounding schemes. Our approach is currently restricted inasmuch as it cannot verify those datapaths where intermediate signals have varying precision (due to rounding). Similarly, saturation arithmetic architectures can also not be verified using our technique. Analysis of such designs requires substantially more work, and is the subject of our future investigations.

\section{CONCLUSIONS}

We have presented a method to determine simulationbounds for equivalence verification of high-level descriptions of arithmetic datapaths. Our approach models the design as a polyfunction from $Z_{2^{n_{1}}} \times Z_{2^{n_{2}}} \times \ldots \times Z_{2^{n} d} \rightarrow Z_{2^{m}}$. Established concepts from number theory and commutative algebra have been analyzed and extended to derive the requisite theoretical results. Practical application of these results was also demonstrated for simulation-based verification. We were able to verify equivalence for a number of circuits by simulating the RTL descriptions. Also, the bugs in the design were detected within the proposed bound. As part of future work, we are investigating applications of the proposed results to various other 
TABLE I

REQUIRED NUMBER OF SIMULATION VECTORS

\begin{tabular}{|c|c|c|c|c|c|}
\hline Benchmark & Specs & Total & Proposed & Required & EQUIVI \\
\hline & Var/Deg $/<n_{1}, \ldots, n_{d}>/ m$ & Test Vectors & Test Vectors & Test Vectors & BUG ? \\
\hline \multicolumn{6}{|l|}{ Fault-free circuits } \\
\hline IRR & $2 / 4 /<12,8>/ 16$ & $2^{20}$ & $18^{2}$ & $18^{2}$ & EQUIV \\
\hline PSK & $2 / 4 /<11,14>/ 16$ & $2^{25}$ & $18^{2}$ & $18^{2}$ & EQUIV \\
\hline Degree-4 filter 1 & $3 / 4 /<15,11,13>/ 16$ & $2^{39}$ & $18^{3}$ & $18^{3}$ & EQUIV \\
\hline Degree-4 filter 2 & $1 / 4 /<12>/ 16$ & $2^{12}$ & 18 & 18 & EQUIV \\
\hline Savitzky-Golay filter & $5 / 3 /<16,16,14,12,8>/ 16$ & $2^{66}$ & $18^{5}$ & $18^{5}$ & EQUIV \\
\hline $4^{\text {th }}$ Order IIR & $2 / 4 /<24,29>/ 32$ & $2^{53}$ & $34^{2}$ & $34^{2}$ & EQUIV \\
\hline MIBENCH & $2 / 9 /<16,12>/ 16$ & $2^{28}$ & $18^{2}$ & $18^{2}$ & EQUIV \\
\hline \multicolumn{6}{|l|}{ Faulty circuits } \\
\hline Anti-alias function & $1 / 6 /<11>/ 16$ & $2^{11}$ & 18 & 18 & BUG \\
\hline Horner Polynomial & $3 / 4 /<10,8,16>/ 16$ & $2^{34}$ & $18^{3}(5832)$ & 4335 & BUG \\
\hline Vanishing polynomial & $2 / 10 /<12,12>/ 16$ & $2^{24}$ & $18^{2}(324)$ & 103 & BUG \\
\hline
\end{tabular}

datapath computations such as those that implement rounding and saturation arithmetic.

\section{REFERENCES}

[1] D. Menard, D Chillet, F Charot, and O. Sentieys, "Automatic Floatingpoint to Fixed-point Conversion for DSP Code Generation", in Intl. Conf. Compiler, Architecture, Synthesis Embedded Sys., CASES, 2002.

[2] I. A. Groute and K. Keane, "M(VH)DL: A MATLAB to VHDL Conversion Toolbox for Digital Control", in IFAC Symp. on Computer-Aided Control System Design, Sept. 2000.

[3] MATLAB/Simulink; Matlab to RTL Translator, http://www.mathworks.com/products/simulink.

[4] A. Peymandoust and G. DeMicheli, "Application of Symbolic Computer Algebra in High-Level Data-Flow Synthesis", IEEE Trans. CAD, vol. 22, pp. 1154-11656, 2003.

[5] S. Gopalakrishnan, P. Kalla, and F. Enescu, "Optimizing Fixed-Size Bit-Vector Arithmetic using Finite-Ring Algebra", in To appear, IWLS, 2006.

[6] D. E. Knuth, The Art of Computer Programming, Vol. II, Seminumerical Algorithms, Addison Wesley, 1998.

[7] Crandall R. and Pomerance C., Prime Numbers: a Computational Perspective, Springer, 2000.

[8] Z. Chen, "On polynomial functions from $\mathrm{Z}_{n_{1}} \times \mathrm{Z}_{n_{2}} \times \cdots \times \mathrm{Z}_{n_{r}}$ to $\mathrm{Z}_{m}$ ", Discrete Math., vol. 162, pp. 67-76, 1996.

[9] J. Smith and G. DeMicheli, "Polynomial methods for component maching and verification", in In Proc. ICCAD, 1998.

[10] P. Sanchez and S. Dey, "Simulation-Based System-Level Verification using Polynomials", in High-Level Design Validation \& Test Workshop, HLDVT, 1999.

[11] I. Ugarte and P. Sanchez, "Formal Meaning of Coverage Metrics in Simulation-Based Hardware Design Verification", in High-Level Design Validation \& Test Workshop, HLDVT, 2005.

[12] N. Shekhar, P. Kalla, Enescu F., and S. Gopalakrishnan, "Exploiting Vanishing Polynomials for Equivalence Verification of Fixed-Size Arithmetic Datapaths", in Intl. Conf. Computer Design, 2005.

[13] N. Shekhar, P. Kalla, and Enescu F., "Equivalence Verification Arithmetic Datapaths with Multiple Word-length Operands", in Design Automation and Test in Europe (DATE), 2006.

[14] T. Raudvere, A. K. Singh, I. Sander, and A. Jantsch, "System level verification of digital signal processing applications based on the polynomial abstraction technique", in Proc. of ICCAD, pp. 285-290, 2005.

[15] J. E. Eaton, "The Fundamental Theorem of Algebra", American Mathematical Monthly, vol. 67, pp. 578-579, 1960.

[16] R. E. Bryant, "A Methodology for Hardware Logic Simulation”, Journal of the ACM, vol. 38, pp. 299-328, 1991.

[17] R. E. Bryant, "Formal Verification of Memory Circuits by Switch-Level Simulation”, IEEE Transactions on CAD, vol. 10, pp. 94-102, Jan, 1991.

[18] R. Ernst and J. Bhasker, "Simulation- Based Verification for High-Level Synthesis", IEEE Design and Test, vol. 8, pp. 14-20, Jan, 1991.

[19] D. Brand, "Exhaustive Simulation Need Not Require an Exponential Number of Tests", in Proc. ICCAD, pp. 98-101, Nov 1992.

[20] E. Clarke, S. German, Y. Lu, H. Veith, and D. Wang, "Executable Protocol Specification in ESL", in Proc. FMCAD, Nov 2000.
[21] J. Yuan, K. Shultz, C. Pixley, H. Miller, and A. Aziz, "Modeling design constraints and biasing in simulation using bdds", in Proc. ICCAD, Nov 1999.

[22] K. Shimizu and D. L. Dill, "Deriving a Simulation Input Generator and a Coverage Metric From a Formal Specification", in Proc. DAC, pp. 801-806, 2002.

[23] K. Shimizu and D. L. Dill, "Using Formal Specifications for Functional Validation of Hardware Designs", IEEE Design \& Test of Computers, vol. 19, pp. 96-106, 2002.

[24] N. Shekhar, P. Kalla, Enescu F., and S. Gopalakrishnan, "Equivalence Verification of Polynomial Datapaths with Fixed-Size Bit-Vectors using Finit Ring Algebra", in Intl. Conf. on Computer-Aided Design, ICCAD, 2005.

[25] J. Moses, "Algebraic simplification: A guide for the perplexed", Comm. ACM, vol. 14, pp. 548-560, 1971.

[26] D. Singmaster, "On Polynomial Functions (mod m)", J. Number Theory, vol. 6, pp. 345-352, 1974.

[27] Z. Chen, "On polynomial functions from $z_{n}$ to $z_{m}$ ", Discrete Math., vol. 137, pp. 137-145, 1995.

[28] N. J. A. Sloane and S. Plouffe, The Encyclopedia of Integer Sequences, Academic Press, 1995.

[29] C. Jordan, Calculus of Finite Differences, New York: Chelsea, 1965.

[30] V. J. Mathews and G. L. Sicuranza, Polynomial Signal Processing, Wiley-Interscience, 2000.

[31] M. R. Guthaus, J. S. Ringenberg, D. Ernst, T. M. Austin, T. Mudge, and R. B. Brown, "Mibench: A Free, Commercially Representative Embedded Benchmark Suite", in IEEE 4th Annual Workshop on Workload Characterization, Dec 2001.

[32] A. K. Verma and P. Ienne, "Improved use of the Carry-save Representation for the Synthesis of Complex Arithmetic Circuits", in Proceedings of the International Conference on Computer Aided Design, 2004.

[33] Universite' de Bretagne Sud LESTER, "Gaut, Architectural Synthesis Tool”, http://lester.univ-ubs.fr:8080, vol. , 2004. 